Chemical Physics 135 (1989) 195-201

North-Holland, Amsterdam

\title{
A UNIFIED THEORY OF RADIATIVE AND RADIATIONLESS MOLECULAR ENERGY TRANSFER
}

\author{
D.L. ANDREWS \\ School of Chemical Sciences, University of East Anglia, Norwich NR4 7TJ, UK
}

Received 9 March 1989

\begin{abstract}
Resonant energy transfer by dipolar coupling is generally regarded as occurring through two distinct mechanisms. One mechanism is radiative transfer, in which a photon is emitted by the donor molecule and is subsequently absorbed by an acceptor species. The other mechanism is mediated by a radiationless Coulombic intermolecular interaction. Whilst both the radiative and radiationless mechanisms require an overlap between the emission spectrum of the donor and the absorption spectrum of the acceptor, distinctions are usually drawn between other mechanistic features. However, by developing a fully quantum electrodynamical treatment of the radiationless process, it can be shown that Förster's result arises as the short-range limit of a more general dipoledipole interaction based on virtual photon coupling. At large separations $R$, retardation effects modify the form of the usual $R^{-6}$ distance dependence to $R^{-2}$, and the result can be identified with the classical result for radiative transfer. Hence the radiative and radiationless mechanisms for energy transfer must be regarded as indistinguishable.
\end{abstract}

\section{Introduction}

The migration of energy by intermolecular energy transfer is a highly significant feature of ultrafast photochemistry in the condensed phase. Donor molecules initially excited by photoabsorption can transfer energy to neighbouring acceptor molecules by a variety of mechanisms. For molecules separated by sub-nanometer distances, a direct energy exchange resulting from wavefunction overlap can occur, and is characterised by a negative exponential dependence on the separation $R$. At larger distances when wavefunction overlap is essentially negligible, the dominant processes for resonant energy transfer are radiative transfer, in which a photon is emitted by the donor and is subsequently absorbed by an acceptor species, and a radiationless Coulombic intermolecular interaction. Both of these processes are normally associated with dipolar coupling, and play a significant role in the dynamics of energy trapping in the photosynthetic unit.

Whilst both of these resonant coupling mechanisms require an overlap between the emission spectrum of the donor and the absorption spectrum of the acceptor, distinctions are usually drawn between other mechanistic features. These have recently been dis- cussed by Bernard et al. [1], and can be summarised in the statement that the radiative mechanism involves the emission and absorption of transverse photons with two independent polarisation components, whilst the Coulombic mechanism is mediated by a longitudinal interaction which does not involve transverse photons. Förster's phenomenological treatment of the radiationless mechanism for energy transfer first provided the now well-known result that for dipolar coupling the rate of transfer has an $R^{-6}$ dependence [2]. A later quantum mechanical treatment, also due to Förster produced the same result [3]. It is now widely accepted that $R^{-6}$ behaviour applies generally, except in cases of large molecules separated by comparatively short distances [4].

It is the purpose of this paper to show that the radiative and radiationless energy transfer mechanisms are, in fact, equivalent. This is demonstrated by a detailed treatment of the two processes using well-established quantum electrodynamical methods [5]. In particular, it is shown that Förster's result arises as the short-range limit of a more general dipole-dipole interaction based on virtual photon coupling. The term "virtual" arises because such photons cannot be observed, their role being similar to that of the virtual molecular states involved in the 
description of scattering processes [6]. At large separations, retardation effects modify the form of the $R^{-6}$ distance dependence to $R^{-2}$, and the result can be identified with the classical result for radiative transfer. Hence the radiative and radiationless mechanisms for energy transfer must be regarded as indistinguishable.

\section{Quantum electrodynamical formulation of the theory}

To begin, the quantum electrodynamical Hamiltonian for a system comprising a single donor molecule (D) and a single acceptor molecule (A), coupled to the radiation field by dipolar coupling, can be written as follows:

$H=H_{\mathrm{mol}}^{\mathrm{D}}+H_{\mathrm{mol}}^{\mathrm{A}}+H_{\mathrm{int}}^{\mathrm{D}}+H_{\text {int }}^{\mathrm{A}}+H_{\mathrm{rad}}$,

where $H_{\mathrm{mol}}^{\mathrm{D}}$ and $H_{\mathrm{mol}}^{\mathrm{A}}$ are the usual unperturbed Schrödinger operators for molecules $\mathrm{D}$ and $\mathrm{A} . H_{\mathrm{int}}^{\mathrm{D}}$ and $H_{\mathrm{int}}^{\mathrm{A}}$ are the dipolar interaction operators

$H_{\mathrm{int}}^{\mathrm{D}}=-\epsilon_{0}^{-1} \mu^{\mathrm{D}} \cdot d^{1}\left(R_{\mathrm{D}}\right)$,

$H_{\mathrm{int}}^{\mathrm{A}}=-\epsilon_{0}^{-1} \mu^{\mathrm{A}} \cdot d^{\perp}\left(R_{\mathrm{A}}\right)$,

and $H_{\text {rad }}$ is the radiation Hamiltonian

$H_{\mathrm{rad}}=\frac{1}{2} \int\left(\epsilon_{0}^{-1} d^{\perp 2}+\epsilon_{0} c^{2} b^{2}\right) \mathrm{d}^{3} r$.

Here $\mu^{\mathrm{D}}, \mu^{\mathrm{A}}$ are the dipole moment operators for molecules $\mathrm{D}, \mathrm{A}$ located at $\boldsymbol{R}_{\mathrm{D}}, \boldsymbol{R}_{\mathrm{A}}$ and $\boldsymbol{b}, \boldsymbol{d}^{\perp}$ represent the magnetic field and transverse electric displacement operators for the radiation field. The latter has the mode expansion

$$
\begin{aligned}
& d^{\perp}(\boldsymbol{r})=\sum_{k, \lambda}\left(\frac{\hbar c k \epsilon_{0}}{2 V}\right)^{1 / 2} \\
& \times \mathrm{i}\left[\boldsymbol{e}^{(\lambda)}(\boldsymbol{k}) a^{(\lambda)}(\boldsymbol{k}) \exp (\mathrm{i} \boldsymbol{k} \cdot \boldsymbol{r})\right. \\
& \left.-\bar{e}^{(\lambda)}(\boldsymbol{k}) a^{+(\lambda)}(\boldsymbol{k}) \exp (-\mathrm{i} \boldsymbol{k} \cdot \boldsymbol{r})\right],
\end{aligned}
$$

where $a^{(\lambda)}(k)$ and $a^{+(\lambda)}(k)$ are respectively the annihilation and creation operators for a radiation mode with wave vector $\boldsymbol{k}$ (frequency $\omega=c k$ ) and polarisation vector $e^{(\lambda)}(k) ; V$ is the quantisation volume.

The probability amplitudes $M_{f}$ for emission and absorption processes can both be derived from the leading first-order term in the time-dependent perturbation series

$$
\begin{aligned}
M_{f i} & =\left\langle f\left|H_{\mathrm{int}}\right| i\right\rangle \\
& +\sum_{r} \frac{\left\langle f\left|H_{\mathrm{int}}\right| r\right\rangle\left\langle r\left|H_{\mathrm{int}}\right| i\right\rangle}{E_{i}-E_{r}}+\ldots,
\end{aligned}
$$

where $|i\rangle$ and $|f\rangle$ denote initial and final states; the probability amplitude for energy transfer mediated by virtual photon exchange results from the secondorder term in eq. (5). The time-ordered diagrams corresponding to each process are shown in fig. 1: (a) and (b) represent emission and absorption, respectively, and both (c) and (d) together contribute to virtual photon exchange. In fig. $1 \mathrm{c}$ for example as time progresses upwards, the initial state of the system is represented by having the donor molecule in an excited state $|m\rangle$ and the acceptor in the ground state $|0\rangle$. Emission of a virtual photon with wave vector $\boldsymbol{\kappa}$ and polarisation vector $\boldsymbol{E}$ then takes place, by means of which the donor returns to its ground state. Finally, the virtual photon is absorbed by the acceptor, which is thereby promoted to the state $|m\rangle$; for simplicity it is assumed that the donor and acceptor are

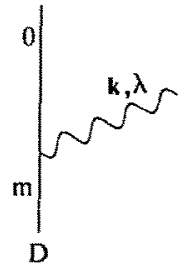

a

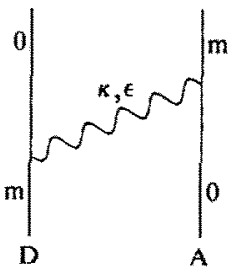

c

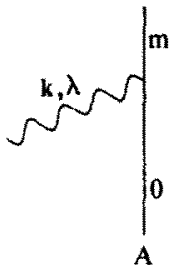

b

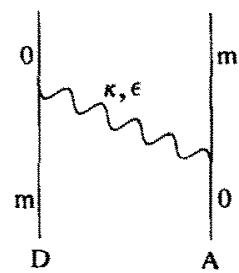

d
Fig. 1. Time-ordered diagrams for resonance energy transfer; (a) and (b) relate to separate donor emission and acceptor absorption processes respectively; (c) and (d) together represent a coupled process mediated by virtual photon exchange. 
chemically equivalent. As always all possible timeorderings need to be included in the calculation, so that there is also a contribution from fig. 1d, in which the virtual photon propagates from $A$ to $D$. The results for the various probability amplitudes are as follows, where $\mu^{m 0(D)}$ for example, represents the transition dipole moment for the transition $|m\rangle \leftarrow|0\rangle$ at D:

$$
\begin{aligned}
& M_{(\mathrm{a})}=\mathrm{i}\left(\frac{\hbar c k}{2 \epsilon_{0} V}\right)^{1 / 2} \mu^{m 0(\mathrm{D})}-\overline{\boldsymbol{e}}^{(\lambda)}(k) \\
& \times \exp \left(-i \boldsymbol{k} \cdot \boldsymbol{R}_{\mathrm{D}}\right), \\
& M_{(\mathrm{b})}=-\mathrm{i}\left(\frac{\hbar c k}{2 \epsilon_{0} V}\right)^{1 / 2} \mu^{0 m(\mathrm{~A})} \cdot e^{(\lambda)}(k) \\
& \times \exp \left(\mathrm{i} \boldsymbol{k} \cdot \boldsymbol{R}_{\mathrm{A}}\right) \text {, } \\
& M_{(\mathrm{c})}=\sum_{\kappa, \lambda} \frac{\kappa}{2 \epsilon_{0} V}\left[\boldsymbol{\mu}^{\left.m 0(D) \cdot \bar{\varepsilon}^{(\lambda)}(\kappa)\right]}\right. \\
& \times\left[\mu^{0 m(A)} \cdot e^{(\lambda)}(\boldsymbol{x})\right](k-\kappa)^{-1} \\
& \times \exp \left[i \kappa \cdot\left(R_{\mathrm{A}}-\boldsymbol{R}_{\mathrm{D}}\right)\right] \text {, } \\
& M_{(\mathrm{d})}=\sum_{\kappa, \lambda} \frac{\kappa}{2 \epsilon_{0} V}\left[\mu^{m 0(\mathrm{D})} \cdot \boldsymbol{\varepsilon}^{(\lambda)}(\boldsymbol{\kappa})\right] \\
& \times\left[\mu^{0 m(\mathrm{~A})} \cdot \overline{\boldsymbol{E}}^{(\lambda)}(\boldsymbol{\kappa})\right](k+\kappa)^{-1} \\
& \times \exp \left[-\mathrm{i} \kappa \cdot\left(\boldsymbol{R}_{\mathrm{A}}-\boldsymbol{R}_{\mathrm{D}}\right)\right] .
\end{aligned}
$$

Here use has been made of the relation

$$
n c k=E_{m}-E_{0}
$$

and there is no restriction on the energy or polarisation of the virtual photon.

The appropriate sums over $\boldsymbol{x}$ and $\lambda$ in eqs. (9) and (10) can be performed using the relation $[7,8]$

$$
\begin{aligned}
\sum_{\boldsymbol{\kappa}, \lambda} & \frac{\boldsymbol{\kappa}}{2 \epsilon_{0} V} \varepsilon_{i}^{(\lambda)}(\boldsymbol{\kappa}) \bar{\varepsilon}^{(\lambda)}(\boldsymbol{\kappa}) \\
& \times\left[(k-\kappa)^{-1} \exp (\mathrm{i} \boldsymbol{\kappa} \cdot \boldsymbol{R})\right. \\
& \left.-(k+\kappa)^{-1} \exp (-\mathrm{i} \boldsymbol{\kappa} \cdot \boldsymbol{R})\right] \\
& =V_{i j}(k, \boldsymbol{R})
\end{aligned}
$$

where $\boldsymbol{R}$ represents the displacement vector $\left(\boldsymbol{R}_{\mathrm{A}}-\boldsymbol{R}_{\mathrm{D}}\right)$ and

$$
V_{i j}(k, R)=\sigma_{i j}+i \tau_{i j}
$$

is a second-rank index-symmetric Cartesian tensor representing the retarded resonance electric dipoleelectric dipole coupling. The functions $\sigma_{i j}, \tau_{i j}$ in eq. (12) are defined by

$$
\begin{aligned}
\sigma_{i j} & =\left(4 \pi \epsilon_{0} R^{3}\right)^{-1} \\
& \times\left[\left(\delta_{i j}-3 \hat{R}_{i} \hat{R}_{j}\right)(\cos k R+k R \sin k R)\right. \\
& \left.-\left(\delta_{i j}-\hat{R}_{i} \hat{R}_{j}\right) k^{2} R^{2} \cos k R\right], \\
\tau_{i j} & =\left(4 \pi \epsilon_{0} R^{3}\right)^{-1} \\
& \times\left[\left(\delta_{i j}-3 \hat{R}_{i} \hat{R}_{j}\right)(k R \cos k R-\sin k R)\right. \\
& \left.-\left(\delta_{i j}-\hat{R}_{i} \hat{R}_{j}\right) k^{2} R^{2} \sin k R\right] .
\end{aligned}
$$

In passing, it is worth noting that the long-range behaviour of both these functions is dominated by the $\delta_{i j}-\hat{R}_{i} \hat{R}_{j}$ terms which are of purely transverse nature with respect to the intermolecular vector $\boldsymbol{R}$. Here the absence of any longitudinal component reflects the disappearance of static terms associated with purely Coulombic interactions. Elsewhere, $\sigma_{i j}$ and $\tau_{i j}$ contain terms of both transverse and longitudinal character. Using the results of eqs. (11)-(14), the total probability amplitude for virtual photon coupling can be written using the implied summation convention for repeated indices as

$M_{(\mathrm{c}+\mathrm{d})}=\mu_{i}^{0 m(\mathrm{~A})} \mu_{j}^{m 0(\mathrm{D})} V_{i j}(k, \boldsymbol{R})$.

With the results of eqs. (6), (7) and (15), the requisite rate equations can now be derived using the Fermi golden rule:

$\Gamma=\frac{2 \pi}{h}|M|^{2} \rho_{f}$,

where $\rho_{f}$ is the appropriate density of final states.

\section{Theory of radiative energy transfer}

The process of interest results in excitation of the acceptor $A$, and it is simplest to begin by deriving an expression for the rate of this excitation in terms of the irradiance (power per unit area) incident upon A. For simplicity it is convenient to assume a random distribution of acceptor orientations, and after substituting eq. (6) into eq. (16) and performing the appropriate three-dimensional rotational averages, we have 
$\Gamma_{\left(\mathrm{A} \rightarrow \mathrm{A}^{*}\right)}=\frac{\pi c k}{3 \epsilon_{0} V}\left|\mu^{0 m(\mathrm{~A})}\right|^{2} \rho_{m}$,

where $\rho_{m}$ is the density of molecular states associated with the energy level $E_{m}$. The calculation leading to eq. (17) involves the assumption of only one photon in the quantisation volume $V$; this corresponds to an irradiance given by

$I=\hbar c^{2} k / V$.

Since there is no restriction on the quantisation volume, eqs. (17) and (18) can be combined to produce a result which is valid for any irradiance and is therefore as follows:

$\Gamma_{\left(\mathrm{A} \rightarrow \mathrm{A}^{*}\right)}=\frac{\pi I}{3 \hbar c \epsilon_{0}}\left|\mu^{0 m(\mathrm{~A})}\right|^{2} \rho_{m}$.

The irradiance of $A$ due to spontaneous emission by the donor $D$ can now be obtained from eqs. (6) and (16) as follows. The rate of emission into an element of solid angle $\mathrm{d} \Omega$ is given by the expression

$\Gamma_{\left(\mathrm{D}^{*} \rightarrow \mathrm{D}\right)}(\mathrm{d} \Omega)=\frac{k^{3}}{12 \pi^{2} \epsilon_{0} h}\left|\mu^{0 m(\mathrm{D})}\right|^{2} \mathrm{~d} \Omega$,

where rotational averaging and summation over orthogonal polarisation components have been effected, and use has been made of the result

$\rho_{\mathrm{rad}}=\frac{k^{2} V \mathrm{~d} \Omega}{8 \pi^{3} \hbar c}$

for the density of radiation states. Integration over $4 \pi$ steradians in eq. (20) leads to the usual result for the Einstein $A$ coefficient. However, here we require the intensity of radiation incident upon the acceptor molecule. This can be obtained by multiplying the rate (20) by $\hbar c k$ for the power, and dividing by the cross sectional area $R^{2} \mathrm{~d} \Omega$; hence

$I=\frac{c k^{4}}{12 \pi^{2} \epsilon_{0} R^{2}}\left|\mu^{0 m(\mathrm{D})}\right|^{2}$

Thus from eqs. (19) and (22) we obtain the following result for the rate of radiative energy transfer

$\Gamma_{\left(\mathrm{D}^{*} \mathrm{~A} \rightarrow \mathrm{DA} \mathrm{A}^{*}\right)}^{\text {radive }}=\frac{k^{4}\left|\mu^{0 m}\right|^{4} \rho_{m}}{36 \pi \hbar \epsilon_{0}^{2} R^{2}}$

where use has been made of the fact that the transi- tion dipole moment has the same magnitude for both $A$ and $D$.

\section{Theory of radiationless energy transfer}

In this case the rate is obtained by substituting the virtual photon coupling matrix element, (15), into the Fermi rule. Once again, after performing the necessary rotational averages, we obtain

$\Gamma_{\substack{\text { non-radiative } \\\left(\mathrm{D}^{*} \rightarrow \mathrm{DA}^{*}\right)}}^{\text {a }}=\frac{2 \pi\left|\mu^{0 m}\right|^{4} \rho_{m}}{9 \hbar} A(k, \boldsymbol{R})$,

where

$$
\begin{aligned}
& A(k, \boldsymbol{R})=V_{i j}(k, \boldsymbol{R}) \bar{V}_{i j}(k, \boldsymbol{R}) \\
& \quad=2\left(4 \pi \epsilon_{0} R^{3}\right)^{-2}\left(3+k^{2} R^{2}+k^{4} R^{4}\right) .
\end{aligned}
$$

Whilst this result is true for all distances, the limiting values for $k R \ll 1$ (the near zone) and for $k R \gg 1$ (the wave zone) are of special interest. In the former case, corresponding to the range of separations over which energy transfer is most significant, only the first term in eq. (25) contributes, and we have

$\left.\Gamma_{\left(\mathrm{D}^{*} \mathrm{~A} \rightarrow \mathrm{DA}^{*}\right)}^{\text {non-adive }}\right)=\frac{\left|\mu^{0 m}\right|^{4} \rho_{m}}{12 \pi \hbar \epsilon_{0}^{2} R^{6}} \quad(k R \ll 1)$.

However, the familiar $R^{-6}$ dependence is lost as $R$ approaches $1 / k$, and in the long range it is the final term in eq. (25) which dominates leading to the result

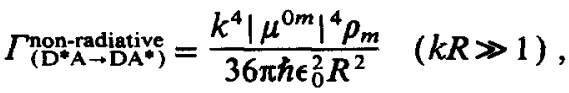

which is identical to the radiative result of eq. (23). The long-range behaviour of the virtual photon is thus identifiable with propagation of a real photon and leads to a molecular analogue of Lambert's inversesquare law. Hence the radiative and radiationless mechanisms for energy transfer must be regarded as indistinguishable.

\section{Excitation transfer function}

As seen above, the new excitation transfer function $A(k, R)$ given by eq. (25) represents a relativistically correct result for dipolar coupling to which the stan- 
dard Förster result is a near-zone approximation. It is interesting to note that a range dependence of similar form, varying with $R^{-n}$ where $2<n<6$, was first suggested on an entirely different basis thirty years ago [9]. Fig. 2 shows a $\log -\log$ plot of $A$ against $R$ for a value of $k=9 \times 10^{6} \mathrm{~m}^{-1}$, corresponding to transfer of the energy associated with photoabsorption at $\approx 700 \mathrm{~nm}$. As such, the curve is therefore appropriate for considering the range dependence of energy transfer within the photosynthetic unit (PSU). The steeper dotted line (slope -6) shows the Förster result as the short-range asymptote; the other dotted line (slope -2 ) shows the radiative transfer rate as the long-range asymptote.

It is evident that departures from Förster behaviour become significant at relatively short distances $R>100 \mathrm{~nm}$. It is surprising that what are essentially relativistic correction effects should make their appearance so soon. Within the PSU, this corresponds to about fifty times the nearest-neighbour distance between chlorophyll units. Beyond this distance, the difference between the true rate and the Förster re-

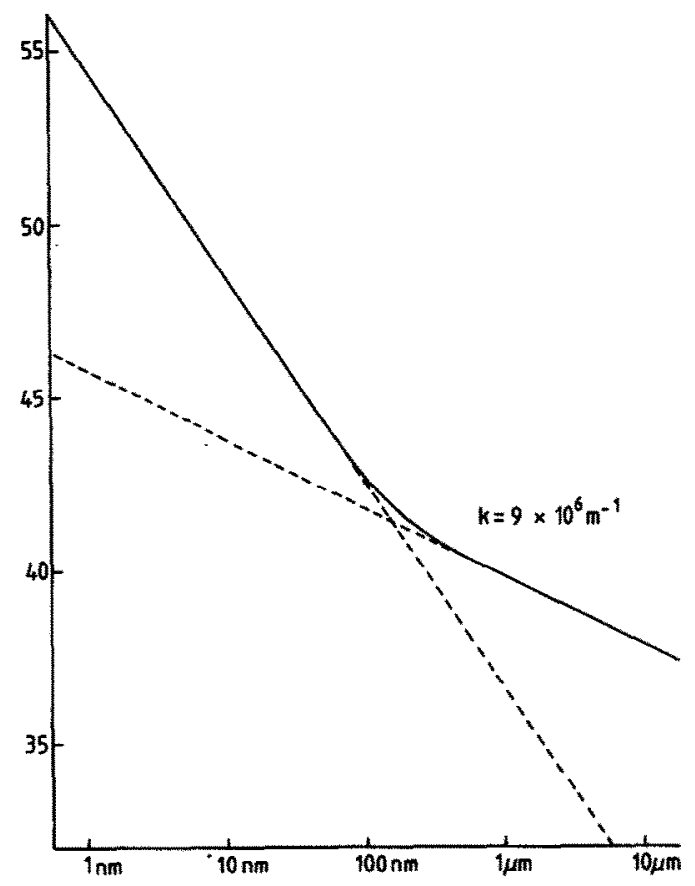

Fig. 2. Typical $\log$-log plot on an arbitrary vertical scale of the excitation transfer function $A(k, R)$ against intermolecular distance. sult rapidly increases in magnitude, the true rate being very much greater than the Förster result implies. Consequently many computational simulations of photosynthetic energy transfer may be leading to rates which are significantly in error.

\section{Critical distances}

The Förster critical distance $R_{0}$ is defined as the distance between a donor and acceptor at which the rate of radiative decay by the donor and the rate of radiationless energy transfer to the acceptor become equal. Standard treatments [10] lead to the following expression for $R_{0}$ :

$R_{0}=\left(\frac{3 f}{4 \pi} \int \frac{c^{4}}{\omega^{4} n(\omega)} F(\omega) \sigma(\omega) \mathrm{d} \omega\right)^{1 / 6}$,

where $f$ is an undetermined orientation factor, $n(\omega)$ is the refractive index of the medium at circular frequency $\omega$, and $F(\omega), \sigma(\omega)$ are the normalised donor fluorescence emission spectrum and acceptor absorption cross section respectively.

In the unified theory presented in this paper, another critical distance arises, corresponding to the point at which the classical rates of radiationless and radiative energy transfer coincide. This distance $R_{0}^{\prime}$ is represented by the point at which the two dotted lines in fig. 2 intersect. The explicit result for $R_{0}^{\prime}$ is obtained by equating the first $\left(R^{-6}\right)$ and third $\left(R^{-2}\right)$ terms in eq. (25), leading to the result

$R_{0}^{\prime}=3^{1 / 4} k^{-1}$.

The evaluation of eq. (28) leads to values of $R_{0}$ typically in the region $5-8 \mathrm{~nm}$, whereas eq. (29) produces a result of $R_{0}^{\prime} \approx 150 \mathrm{~nm}$. The reason for this dramatic difference is as follows.

Whilst the necessity for an overlap between the donor fluorescence and acceptor absorption spectra is explicit in eq. (28), it is implicit in the theory leading to eq. (29). However, the physical implications are different, in that the unified theory produces a dependence on the overlap integral which is identical for both the radiative and radiationless mechanisms; similar remarks apply to the orientation factor [11]. Thus in both cases the observed rates will result from 
integration of eqs. (23) and (24) over the appropriate emission bandwidth.

\section{Synergistic effects in the absorption of white light}

Recent studies have shown that virtual photon coupling may give rise to novel effects in the absorption of very intense white light such as that produced by self-phase modulation of mode-locked laser light [12]. This type of source has been widely used to monitor the primary processes of photosynthesis [13]. There are two mechanisms by which the interaction of photoreceptor molecules may produce synergistic absorption effects, as shown in fig. 3. Although two photons $\hbar \omega_{1}$ and $h \omega_{2}$ are absorbed and two acceptor molecules $A_{1}$ and $A_{2}$ become excited, the overall process

$\mathrm{A}_{1}+\mathrm{A}_{2}+\hbar \omega_{1}+h \omega_{2} \rightarrow \mathrm{A}_{1}^{*}+\mathrm{A}_{2}^{*}$

can occur even when the photon frequencies lie outside any absorption band of $A_{1}$ or $A_{2}$, provided overall energy conservation is satisfied. In the cooperative mechanism (a) each acceptor molecule absorbs one photon and a virtual photon propagates the en-

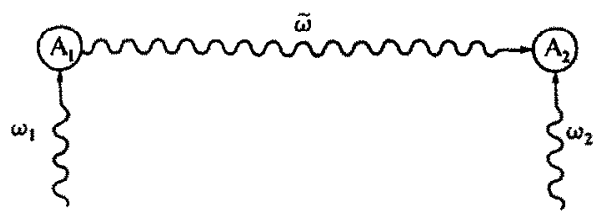

(a)

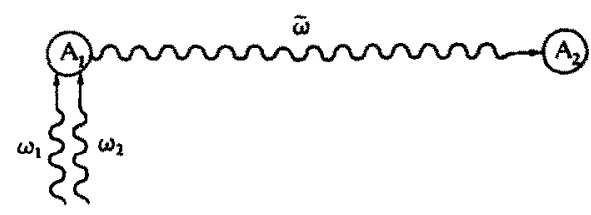

(b)

Fig. 3. Two mechanisms for synergistic absorption of photons, $\omega_{1}, \omega_{2}$; (a) shows the cooperative mechanism and (b) the distributive mechanism. In each case the energy mismatch is mediated by a virtual photon $\bar{\omega}$. ergy mismatch between the two centres; in the distributive mechanism (b) one acceptor absorbs both photons, and the virtual photon conveys the entire excitation energy to the second acceptor.

In the case where there is an isotropic distribution of acceptor molecules, the detailed theory [14,15] shows that the rate of synergistic photoabsorption once more involves the excitation transfer function $A(k, R)$. However, one of the main differences between the cooperative and distributive mechanisms lies in the range over which the limiting near-zone $\left(R^{-6}\right)$ behaviour occurs. The extent of the near-zone for the distributive case is much shorter, with limiting far-zone $R^{-2}$ behaviour already established at $R \approx 1 \mu \mathrm{m}$; for the cooperative case far-zone behaviour typically obtains at $R \approx 10 \mu \mathrm{m}$. The result of this difference is that the long-range rates (which vary with $k^{4}$ ) differ by a factor of $(20)^{4}=160000$ in favour of the distributive mechanism [16].

\section{Conclusion}

It has been shown that a single excitation transfer function $A(k, R)$ provides a unified description of both radiative and radiationless molecular energy transfer. The formulation of the theory is based on virtual photon coupling, and the radiative result corresponds to the long-range case where the photon loses its virtual character. The usual Förster result, which is identified with the short-range limit of $A(k, R)$, is shown to become inaccurate at distances typically around $100 \mathrm{~nm}$, essentially due to relativistic retardation effects, and the unified theory produces dramatically different results for the critical distance. Finally the universal nature of the function $A(k, R)$ is demonstrated by its occurrence in the rate equations for synergistic photoabsorption.

\section{Acknowledgement}

I very gratefully acknowledge stimulating discussions and many useful comments on various parts of this work from B.S. Sherborne and N.P. Blake. 


\section{References}

[1] J.E. Bernard, D.E. Berry and F. Williams, in: Energy Transfer Processes in Condensed Matter, ed. B. Di Bartolo (Plenum Press, New York, 1984) p. 1.

[2] T. Förster, Z. Naturforsch. 4a (1949) 321.

[3] T. Förster, US At. Energy Comm. Div. Biol. Mech. Bull. 18 (1965).

[4] J.C. Chang, J. Chem. Phys. 67 (1977) 3901.

[5] D.P. Craig and T. Thirunamachandran, Molecular Quantum Electrodynamics (Academic Press, New York, 1984).

[6] R.P. Feynman, QED (Princeton Univ. Press., Princeton, 1985).

[7] D.L. Andrews and B.S. Sherborne, J. Chem. Phys. 86 (1987) 4011.

[8] E.A. Power and T. Thirunamachandran, Phys. Rev. A 28 (1983) 2671
[9] A. Gotebiewski and A. Witkowski, Roczniki Chemii 33 (1959) 1443.

[10] C.E. Swenberg, in: Biological Events Probed by Ultrafast Laser Spectroscopy, ed. R.R. Alfano (Academic Press, New York, 1982) p.193.

[11] M. Berberan-Santos and M.J.E. Prieto, J. Chem. Phys. 88 (1988) 6341.

[12] D.L. Andrews, Phys. Rev. A 38 (1988) 5129.

[13] G.R. Fleming, Chemical Applications of Ultrafast Spectroscopy (Oxford Univ. Press, Oxford, 1986) p. 80.

[14] D.L. Andrews and K.P. Hopkins, J. Chem. Phys. 86 (1987) 2453.

[15] D.L. Andrews and K.P. Hopkins, J. Chem. Phys. 88 (1988) 4461.

[16] D.L. Andrews, to be published. 